\title{
Early Experiences with Management of Extra Hepatic Portal Vein Obstruction.
}

Sushil Bahadur Rawal', Srijan Malla ${ }^{1}$, Naresh Kumar Giri ${ }^{1}$, Jyoti Rayamajhi ${ }^{1}$, Kunda Shah ${ }^{1}$.

${ }^{1}$ Department of Surgery, Shree Birendra Hospital.

\begin{abstract}
Introduction: Extrahepatic portal vein obstruction (EHPVO), a rare condition, is increasingly being diagnosed due to easier availability of proper diagnostic tools and early referrals. Here we report our series of 16 cases of EHPVO who were treated at our hospital. The aim of this paper is to share our early experiences of managing this rare and difficult condition. Methods: A retrospective analysis of patients' records admitted with the diagnosis of EHPVO for a period of 18 months from Jan 2008 to June 2010 was performed. Patients were followed up for at least 9 months, their demographic data and outcome of surgery was analysed.

Results: Of the 10 cases fulfilling the inclusion criteria, 9 underwent surgical therapy. Distal lienorenal shunt was performed in 4 patients, proximal lienorenal shunt in 2 patients and devascularisation procedure in 3 patients. Rebleeding was seen in 2 patients. The prognosis of EHPVO was good in patients without a severe underlying disease at a median follow up of 9 months. Conclusion: Conventional surgical therapies have a good outcome in management of EHPVO in our context.
\end{abstract}

Keywords: EHPVO, devascularisation, lienorenal shunt

\section{INTRODUCTION}

Extrahepatic portal vein obstruction (EHPVO) is conventionally defined as obstruction in the pre-hepatic portion of the portal vein and is a relatively uncommon disease in Western countries. Webb and Sherlock ${ }^{1}$ documented 97 cases seen in 18 years at the Royal Free Hospital, London. By contrast, EHPVO is very common in India. At the All India Institute Medical Sciences, 87 cases of EHO Non-cirrhotic portal hypertension and 83 cases of NCPF (Non Cirrhotic portal fibrosis) were treated in 6 years. In Chandigarh, 100 cases of EHO and 38 cases of NCPF were seen in an unspecified period ${ }^{2}$. In Japan, EHO is less common than Idiopathic Portal Hypertension (IPH). The incidence of EHO among 247728 autopsies performed in 1975-1982 was $0.055 \%$. The IPH Study Group of Japan studied 184 surgically and angiographically confirmed cases of EHO in comparison with 469 cases of IPH $\mathrm{IP}^{3}$. Though the exact incidence in our country is not known, there has been increase in the number of patients presenting to our hospital these days. This can be attributed to the easier availability of proper diagnostic tools and early referral. The purpose of this study was to analyze the patients with EHPVO and compare with the published literature.

\section{METHODS}

A retrospective analysis of patients' records, who were admitted with the diagnosis of EHPVO for a period of 18 months from Jan 2008 to June 2010, was performed. The demographic data, clinical presentation, methods of treatment and present status were analyzed. Patients who had complete recorded document and a confirmatory radiological diagnosis (CT and Spleenoportogram) were included in this series. Patients with incomplete documentations and not willing to come for follow up were excluded. Descriptive data analysis was done using Microsoft excel 2010.

\section{RESULTS}

Among 16 provisionally diagnosed cases of EHPVO, only 10 case records fulfilled the inclusion criteria. The age of the patients ranged from 7 years to 26 years with mean age of 12 years. Of these, 4 were male and 6 female. The most common clinical symptom was abdominal pain $(90 \%)$ in combination with diarrhoea and vomiting followed by anorexia. Variceal bleeding was present in 8 patients (80\%), while 6 patients (60\%) presented with features of hypersplenism. None of the patients had features of portal biliopathy. Laboratory signs were non-

\section{Correspondence:}

Lt. Col. Dr. Sushil Bahadur Rawal

Department of Surgery, Shree Birendra Hospital.

Kathmandu, Nepal.

Email: sushilrawal85@yahoo.com

Phone: +977-9851002543 
specific and diagnosis was usually made on USG and CT spleenoportogram (80\%). Prothrombotic states as causative factors were not seen in any of the cases; however in 1 case $(10 \%)$, an infective cause was identified.

Out of 10 patients, 9 underwent surgical intervention for portal hypertension. The remaining one patient with portal vein thrombosis was diagnosed with hemobilia so he underwent left hepatectomy. Distal lienorenal shunt was the most common surgery performed in 4 patients. Devascularisation procedure was performed in 3 patients, of which, two were combined with oesophageal transaction. Two patients underwent proximal lineorenal shunt. Post operative complication of gastrointestinal bleed seen in 2 cases. Upper GI endoscopy revealed esophageal varices which was managed with rubber band ligation. The prognosis of EHPVO remained good in patients without a severe underlying disease (median follow up of 9 months).

\section{DISCUSSION}

Surgery even in the era of liver transplant has an important role to play for EHPVO in developing nation where access to advance care and facility till date is a distant thought. Our understanding of the etiology, natural history, and treatment options for extrahepatic PVT has improved over the last few years. In developing nations, with a scarcity of advanced medical centers, there is invariably a delay in the diagnosis and management of these patients. Although the exact incidence in our country is not known, the introduction of better diagnostic modalities in the recent past has led to an increased detection of this condition. Patients who eluded diagnosis before have now become treatable and surgeries for this condition are being performed more frequently. However there is scanty literature published in Nepal addressing this condition.

Only 9 cases were operated upon over a period of 18 months. If we consider 5 lakh patients affiliated to the military hospital the incidence is very high when compared to studies published from India and other countries. ${ }^{5,6,7,9}$ A further and detailed long term studies is necessary to comment on the incidence. In our series the age of the patient ranged from 7 years to 26 years with a mean age of 12 years. 4 were male and 6 were female with a male to female ratio of 2:3. The age and sex distribution was similar to other published literatures from the Indian subcontinent. ${ }^{6,9}$ The most common clinical symptom in our series was abdominal pain $(90 \%)$ in combination with diarrhoea and vomiting followed by features of GI bleeding (80\%). Laboratory signs were non-specific and diagnosis was usually made by computed tomography spleenoportogram $(80 \%)$. Generally, ultrasonographic examination, preferably with color Doppler capability, is helpful in diagnosis of
EHPVO. When the ultrasonogram is of suboptimal quality or when shunt surgery is being considered then, further investigations such as MR angiography, CT portogram, or angiography are performed. ${ }^{8}$ Spleenoportography is safe, simple, and easily performed. However, the invasive nature of the procedure and dilution of the contrast due to diversion through collateral vessels has markedly limited its use. Contrast-enhanced CT is also highly specific, is less operator dependent, and circumvents the obstacle presented by bowel gas. MR angiography is a noninvasive technique wherein images can be postprocessed to create a projection portal venogram. Moreover, it is possible to tag flowing blood with a radiofrequency pulse to determine flow direction and velocity. Prothrombotic states as causative factors were not seen in any of the cases, however in $1(10 \%)$ case a possible infective cause was identified as the patient also had liver abscess.

A total of 9 patients underwent surgical intervention for portal hypertension. Four cases underwent distal lienorenal shunt, three underwent devascularisation procedurestwo of them combined with esophageal transection and two underwent proximal lieno-renal shunt. Surgical procedures are broadly of two types: portosystemic shunts and ablative procedures. Generally a selective shunt is preferable and a distal lienorenal shunt is the most popular. An overall patency rate of $92 \%$ at median 5 -year followup was reported with selective shunts by Warren et al. ${ }^{7}$ Rebreeding occurred in $12 \%$, shunt dysfunction in $25 \%$, and encephalopathy in none. Several authors have reported good success rate, and a rebleeding rate as low as $4 \%$. Recently the introduction of meso Rex shunt has raised a possible paradigm shift in the therapeutic approach to the management of $\mathrm{EHPVO}^{10}$. Lautz et al recently compared meso Rex shunt with Distal lieno renal shunt and found both procedures effectively relieved symptoms of portal hypertensive bleeding in children with EHPVO, although the meso-Rex better relieves hypersplenism. By restoring normal portal venous circulation, the meso-Rex bypass has additional metabolic benefits. ${ }^{11}$

Devascularisation procedures are usually performed when shuntable veins are not available, in failed shunt surgery or in an emergency when shunt cannot be performed. ${ }^{3}$ In our series, devascularisation was performed in cases with extensive portal vein thrombosis extending into splenic vein and in nonshuntable veins.

\section{CONCLUSIONS}

EHPVO is being frequently detected in our country and even the conventional treatment modalities have good outcome. 


\section{REFERENCES}

1. Webb LJ, Sherlock S. The aetiology, presentation and natural history of extrahepatic portal venous obstruction. Quart J Med NS. 1979;48:627-39.

2. Koshy A. Relationship between NCPF and EHO. In: Okuda K, Omata M, eds. Idiopathic Portal Hypertension.Tokyo: University Tokyo Press, 1983;13-17.

3. Kameda $H$, Yamazaki K, Imai $F$ et al. Obliterative portal venopathy: a comparative study of 184 cases of extrahepatic portal obstruction and 468 cases of idiopathicportal hypertension. J Gastroenterol Hepatol. 1986;1:139-49.

4. Ohnishi K, Okuda K, Ohtsuki T et al. Formation of hilar collaterals or cavernous transformation after portal vein obstruction by hepatocellular carcinoma. Observation in ten patients. Gastroenterology. 1984;87:1150-

5. Mitra SK, Kumar V, Dutta DV, et al. Extrahepatic portal hypertension: a review of 70 cases. J Pediatr Surg. 1978;13:51-54.
6. Sarin SK, Agarwal SR. Extrahepatic Portal Vein Obstruction. Seminars in Liver Disease 2012;22 (1):43-58

7. Warren WD, Henderson JM, Millikan WJ, et al. Management of variceal bleeding in patients with noncirrhotic portal vein thrombosis. Ann Surg. 1988;207:623-34.

8. Maksoud JG, Miles S. Distal splenorenal shunt in children. Ann Surg. 1982;195:401-5.

9. Guharay BN, Sain P, Ramerjee D, et al. Direct splenocaval shunt for selective decompression of portal hypertension in children. Surgery. 1980;87:271-9.

10. Superina $R$, Shneider B, Emre $S$ et al. Surgical guidelines for the management of extra-hepatic portal vein obstruction. Pediatr Transplantation. 2006:10:908-13.

11. Lautz TB, Keys LA, Melvin JC, Ito J, Superina RA. Advantages of the Meso-Rex Bypass Compared with Portosystemic Shunts in the Management of Extrahepatic Portal Vein Obstruction in Children. J Am Coll Surg. 2013;216:83-89. 